\title{
Fractal Substructure of a Nanopowder
}

\author{
Thomas Schwager, ${ }^{1}$ Dietrich E. Wolf, ${ }^{2}$ and Thorsten Pöschel ${ }^{3}$ \\ ${ }^{1}$ Charité, Augustenburger Platz 1, 13353 Berlin, Germany \\ ${ }^{2}$ Universität Duisburg-Essen, Fachbereich Physik, 47048 Duisburg, Germany \\ ${ }^{3}$ Universität Bayreuth, Physikalisches Institut, 95440 Bayreuth, Germany
}

(Dated: October 31, 2018)

\begin{abstract}
The structural evolution of a nano-powder by repeated dispersion and settling can lead to characteristic fractal substructures. This is shown by numerical simulations of a two-dimensional model agglomerate of adhesive rigid particles. The agglomerate is cut into fragments of a characteristic size $\ell$, which then are settling under gravity. Repeating this procedure converges to a loosely packed structure, the properties of which are investigated: a) The final packing density is independent of the initialization, b) the short-range correlation function is independent of the fragment size, c) the structure is fractal up to the fragmentation scale $\ell$ with a fractal dimension close to 1.7 , and d) the relaxation time increases linearly with $\ell$.

PACS numbers: 45.70.-n,45.70.Qj,61.43.Gt,61.43.Hv
\end{abstract}

The van-der-Waals attraction between nano-particles is much stronger than their weight. This is the reason why they agglomerate into ramified, often fractal structures [1]. A well studied example are the agglomerates formed in a filter that collects nano-particles. Once the particle deposits have grown to micrometer size, they can be shaken off the filter fibers easily. Collecting these rather large aerosol flakes in a container leads to what is commonly called a nano-powder, a fragile assembly of partly sintered micrometer flakes made of nano-particles. Depending on the agglomeration process in the aerosol, as well as the influence of diffusion on the deposition process, the nano-powder will have fractal substructures [2]. However it may be questioned, whether these are robust: Shaking, pouring, stirring, and all kinds of random treatments of the container will break the nano-powder up into fragments, presumably with a typical size determined by the prevailing shear forces and much larger than the primary nano-particles, but not necessarily larger than the originally collected aerosol flakes. When allowed to settle, these fragments will reagglomerate, until the next perturbation breaks the nano-powder up again.

Nano-powders can be enormously porous. Porosities of more than $90 \%$ are common. Since many physical properties, such as electrical conductivity, mechanical stability, or catalytic activity, are determined by the structure of the powder, it is important to know, whether there emerge robust generic structural features as a result of repeated fragmentation and reagglomeration processes. In this paper we present large scale simulation results for a simple two-dimensional model which shows such a development of a robust asymptotic structure.

In our model the nano-particles are represented by up to 3 million discs with a narrow size distribution $(10 \%$ variance). As initial state we take a densely packed agglomerate. Below we will show that the final structure is independent of the initial configuration. Then the following procedure is repeated many times: First the ag- glomerate is cut with a square mesh into portions. The linear mesh size $\ell$ can be viewed as the typical scale of the fragmentation process. A portion may consist of several disconnected fragments. These fragment flakes then settle as rigid bodies under gravity without taking adhesion forces with other particles into account. This is justified, if the flakes are sufficiently large, so that their weight exceeds the van-der-Waals force between the nano-particles 10]. Brownian motion is neglected for the same reason. After this reassembly of the fragments the agglomerate is cut again with the square mesh, and so on, see Fig. 1.

The only model parameter is the linear mesh size $\ell$. Note that the limit of small $\ell(\ell \approx$ particle diameter $)$ corresponds to non-cohesive primary particles. In any case, the cohesion forces are assumed to be weak compared to the fragment weight, but strong enough to assure the internal stability of the flakes.

Algorithm: To obtain statistically significant results for the structure of nano-powders one has to consider systems with more than one million particles for many fragmentation-reagglomeration cycles. This is beyond the capability of Molecular Dynamics simulations. For our purpose we therefore generalized a model by Visscher and Bolsterli [3] originally intended for the sequential deposition of macroscopic spherical particles (see [4, 5, 6, 7, 8] for other applications): Each particle starts at a random position well above the already deposited material (the configuration of which is regarded as frozen in). Following gravity, it moves downwards until it touches the bottom of the container, where it sticks, or contacts another already deposited particle. In the latter case it moves, again following gravity, on the surface of the deposit until it either touches the bottom or finds a stable position in contact with the walls and/or previously deposited particles (for more details see [9]).

We generalized this algorithm in order to apply it to the fragments of a nano-powder. In each iteration step we inspect the portions which are cut out by the square 


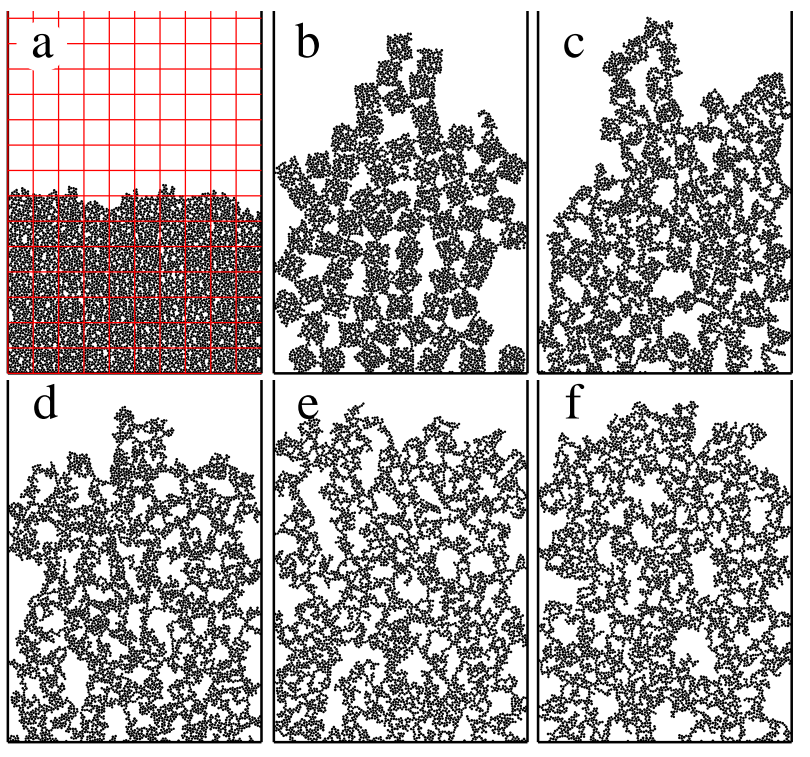

FIG. 1: Evolution of the packing of a nano-powder as described in the text. a) Initial packing generated by random sequential sedimentation [3]. The packing is cut by a square mesh into fragments $(\ell=20)$; b) The fragments are considered as rigid bodies and deposited ( $1^{\text {st }}$ generation). Again the packing is cut by a square mesh (here not shown); c) the fragments are deposited again ( $2^{\text {nd }}$ generation), and so on; $\mathrm{d}$ ) $3^{\text {rd }}$ generation; e) $4^{\text {th }}$ generation; f) $120^{\text {th }}$ generation.

lattice, Fig. 1] with respect to their connectivity. A portion may decompose into several fragments (i.e. clusters of connected nano-particles). We deposit these fragments in a random sequence in the same way as described above. A fragment rolls down the surface of the deposit until the vertical projection of its center of mass falls in between two points of contact. As in the original algorithm, inertia is neglected, which in contrast to previous applications is less of a problem here, because the dynamics of nano-particle flakes is usually strongly damped. In the following, lengths are given in units of the average particle radius, masses in units of the particle mass, and time as number of fragmentation-reagglomeration cycles.

Asymptotic filling height. The original VisscherBolsterly algorithm produces random dense packings of spheres without fractal substructures. Correspondingly, our generalization produces a packing of fragments that is homogeneous on scales larger than the fragmentation length $\ell$, as can be seen in Fig. 11. Surprisingly, however, the short range structure up to size $\ell$ develops robust fractal properties. A first indication is given by the $\ell$ dependence of the filling height.

Starting from a random dense packing of primary particles the filling height increases towards a saturation value. Asymptotically, the powder adopts a very porous, statistically invariant structure, which is robust with respect to fragmentation at a fixed scale and subsequent gravitational settling of the fragments. Remarkably the

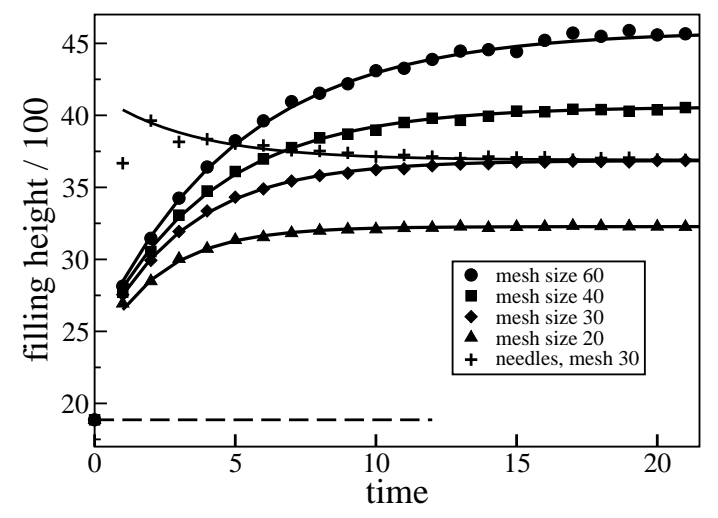

FIG. 2: Evolution of filling height starting from a random dense packing of height $h_{0}$ (indicated by the dashed line). The same asymptotic filling height is reached from above, if the particles initially form a single vertical needle (data marked by + ) instead of a random dense packing. The full lines are fits according to Eq. (1).

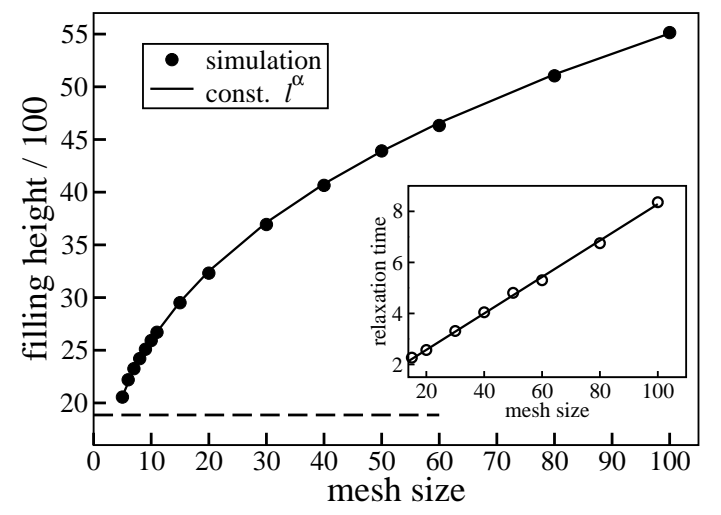

FIG. 3: The asymptotic filling heigth $h_{\infty}$ grows as a power law $h_{\infty}(\ell) \sim \ell^{\alpha}$ with mesh size $\ell$. The full line shows the best fit, $\alpha=0.327$. Inset: The relaxation time $n_{\mathrm{c}}(\ell)$ increases linearly with mesh size.

asymptotic filling height does not depend on the initial configuration: Starting with all particles arranged in a single vertical needle-like chain leads to the same value (see Fig. 2). Except for the first point (the initial condition) the filling height $h_{n}$ at iteration step $n$ can be fitted by an exponential approach of the asymptotic height, $h_{\infty}$, with a relaxation time, $n_{\mathrm{c}}$,

$$
h_{n}=h_{\infty}(\ell)-\left(h_{\infty}(\ell)-h_{0}\right) \exp \left[-n / n_{\mathrm{c}}(\ell)\right] .
$$

The inset of Fig. 3 shows that

$$
n_{\mathrm{c}}(\ell) \propto \ell^{z} \quad \text { with } \quad z=1 .
$$

For the asymptotic filling height, a power law

$$
h_{\infty}(\ell) \propto \ell^{\alpha} \quad \text { with } \quad \alpha=0.327
$$

gives a very good fit(see Fig. [3).

This implies that the number of portions cut from the steady state configuration of a system of width $L$ scales 


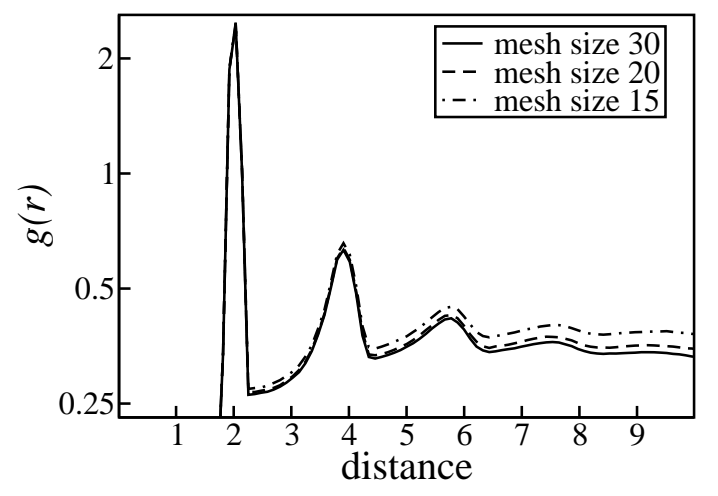

FIG. 4: Pair correlation function (semi-log-plot) of the steady state structure.

like $N_{\mathrm{p}}=h_{\infty} L / \ell^{2} \propto \ell^{\alpha-2}$. Consequently, the mass per portion is $M / N_{\mathrm{p}} \propto \ell^{d_{\mathrm{f}}}$ with

$$
d_{\mathrm{f}}=2-\alpha=1.67 \pm 0.03 .
$$

One can interpret $d_{\mathrm{f}}$ as the fractal dimension of the structure on length scales smaller than the fragmentation length $\ell$, because the short range part of the pair correlation function $g(r)$ does not depend on $\ell$ (see Fig. (4). This means that the short range structure is independent of the overall density. For large distances, on the other hand, the pair correlation function approaches the overall density, which decreases with increasing $\ell$.

On first glance, however, the fractal dimension Eq. (4) seems at odds with the fact, that the asymptotic average fragment mass grows linearly with the mesh size $\ell$ (Fig. 55), suggesting, instead, that the fragments are effectively one-dimensional structures. This puzzle can only be resolved by assuming that the number of disconnected fragments $N_{\mathrm{f}}$ per mesh cell has itself a power law dependence on $\ell$ :

$$
\frac{N_{\mathrm{f}}}{N_{\mathrm{p}}} \propto \ell^{\beta}
$$

As we are going to prove in a moment, the fragmentation-reagglomeration dynamics implies that the exponents $\beta$ and $d_{\mathrm{f}}$ must be related by

$$
\beta=d_{\mathrm{f}}-1 \text {. }
$$

Consequently the mass per fragment is linear in $\ell$ :

$$
\frac{M}{N_{\mathrm{f}}} \propto \frac{\ell^{d_{\mathrm{f}}}}{\ell^{\beta}} \propto \ell .
$$

The proof of the scaling relation Eq. (6) is based on the steady state condition that the deposition of fragments reestablishes on average as many contacts as were cut in the preceding fragmentation step. An $\ell \times \ell-$ mesh cell contains $\propto \ell^{d_{\mathrm{f}}}$ particles, of which $\propto \ell^{d_{\mathrm{f}}-1}$ are at the cell boundary. Hence, the number of particle contacts cut by the boundary of one cell scales as

$$
N_{\text {cut }} \propto \ell^{d_{\mathrm{f}}-1} .
$$

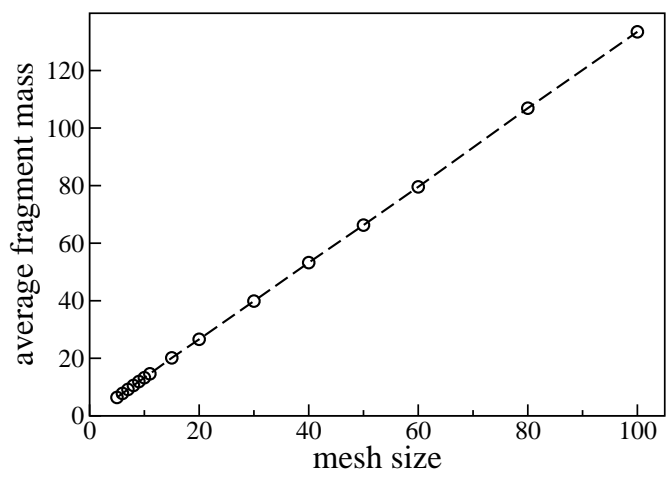

FIG. 5: Average fragment mass as a function of the mesh size $\ell$ in the asymptotic steady state.

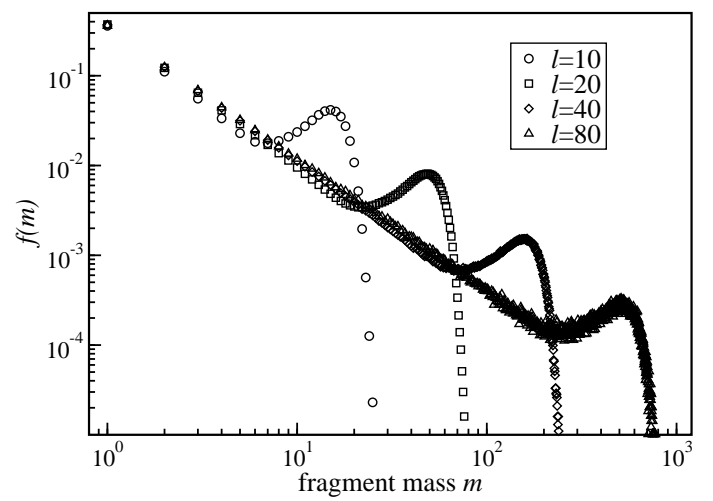

FIG. 6: Normalized fragment mass distribution for different mesh sizes $\ell$. $f(m)$ is the number of fragments of mass $m$ divided by the total number of fragments for a given $\ell$.

Each of the $\ell^{\beta}$ fragments forms two new contacts when deposited. Equating $N_{\text {cut }}=2 N_{\mathrm{f}} / N_{\mathrm{p}}$ gives Eq. (6).

Fragment mass distribution. A detailled understanding of the fragment properties is provided by the distribution of fragment masses, shown in Fig. 6. It reveals that that one must distinguish two types of fragments, large chunks at the upper end of the mass spectrum with a characteristic size $m_{\mathrm{c}}$, and scale invariant dust responsible for the power law part that is cut off by $m_{\mathrm{c}}$. Comparing the mass distributions for different mesh sizes $\ell$ shows, that they can approximately be written in the form

$$
f(m, \ell)=m^{-\tau} \tilde{f}\left(\frac{m}{m_{\mathrm{c}}(\ell)}\right)
$$

where the scaling function $\tilde{f}(x)$ is constant for $x \ll 1$, goes through a maximum at $x=1$, and has an approximately Gaussian tail for $x \gg 1$. The typical mass $m_{\mathrm{c}}$ of the chunks has a power-law dependence on the mesh size, $m_{\mathrm{c}}=0.304 \ell^{1.695}$ (Fig. 7), the exponent being in good agreement with the value of $d_{\mathrm{f}}$, Eq. (4).

The evaluation of the dust exponent, $\tau$, is more difficult, since the slope fitted to the power law part of the 


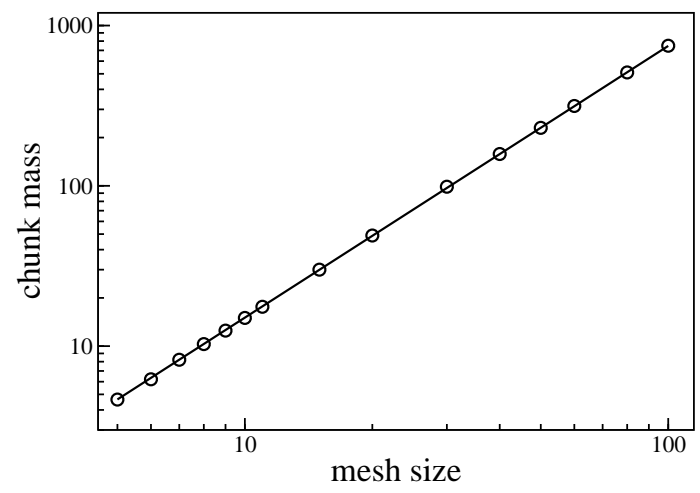

FIG. 7: Chunk mass $m_{c}$ as a function of mesh size. Slope of straight line is 1.695 .

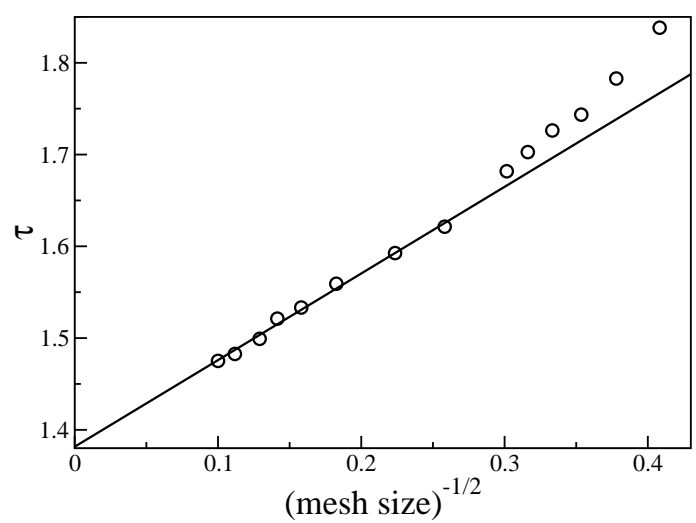

FIG. 8: Effective dust exponent $\tau$ vs. $1 / \ell$.

mass distribution in the log-log-plot Fig. 6 decreases significantly with increasing $\ell$. An extrapolation of $\tau$ for $\ell^{-1 / 2} \rightarrow 0$, see Fig. 8, gives an estimate of $\tau \approx 1.38$. However, an independent method of determining $\tau$ gives a larger value. It is based on the important observation that the width of the chunk-distribution is proportional to $m_{\mathrm{c}}$. Hence the fraction of chunks among the fragments vanishes like

$$
f_{\text {chunks }} \propto m_{\mathrm{c}}^{1-\tau} \propto \ell^{d_{\mathrm{f}}(1-\tau)},
$$

because $\tau$ is larger than 1 . Hence, the normalization of $f(m)$ for $\ell \rightarrow \infty$ implies that

$$
1=f(1) \sum_{m} m^{-\tau}=f(1) \zeta(\tau)
$$

with Riemann's zeta-function at the argument $\tau$. Solving this equation numerically with $f(1) \approx 0.36$ (for the $\ell$ values we considered) gives an estimate $\tau \approx 1.46$.

We have seen, that the overwhelming number of fragments are dust particles, apart from a vanishing fraction of chunks. However, these dust particles carry only a vanishing fraction of the total mass $M$. According to
Eq. (7),

$$
m_{\text {dust }} \leq f(1) \zeta(\tau-1) \frac{N_{\mathrm{f}}}{M} \propto \frac{1}{\ell}
$$

vanishes for $\ell \rightarrow \infty$. Essentially all the mass is in the few chunks. This explains, why the mass (essentially mass of chunks) per fragment (essentially per dust particle) has nothing to do with the fractal dimension.

Now a consistent picture has formed: Each portion (or mesh cell) contains typically one chunk. The number of fragments per portion, which according to Eq. (5) scales like $\ell^{\beta}$, can thus be identified with $1 / f_{\text {chunks }}$, which according to Eq. (10) scales as $\ell^{d_{\mathrm{f}}(\tau-1)}$. This shows that the fractal dimension of the chunks and the dust exponent are not independent of each other. Using Eq. (6) they obey the scaling relation

$$
d_{\mathrm{f}}(2-\tau)=1 .
$$

For $d_{\mathrm{f}}=1.695$ this implies $\tau=1.41$, in between the two $\tau$-values obtained above.

We would like to thank I. Goldhirsch for fruitful discussions. This work was supported by the German-Israeli Foundation by grant no. I-795-166.10/2003.

[1] P. Meakin, in Phase Transitions and Critical Phenomena, edited by C. Domb and J. L. Lebowitz (Academic Press, London, 1988), vol. 12, p. 336.

[2] L. Mädler, A. A. Lall, and S. K. Friedlander, Nanotechnology 17, 4783 (2006).

[3] W. M. Visscher and M. Bolsterli, Nature 239, 504 (1972).

[4] R. Jullien, P. Meakin, and A. Pavlovitch, Europhys. Lett. 22, 523 (1993).

[5] R. Jullien and P. Meakin, Europhys. Lett. 4, 1385 (1987).

[6] R. Jullien and P. Meakin, Europhys. Lett. 6, 629 (1988).

[7] G. Baumann, E. Jobs, and D. E. Wolf, Fractals 1, 767 (1993).

[8] G. Baumann, I. M. Jánosi, and D. E. Wolf, Phys. Rev. E 51, 1879 (1995).

[9] T. Pöschel and T. Schwager, Computational Granular Dynamics: Models and Algorithms (Springer, Berlin, Heidelberg, New-York, 2005).

[10] Occasionally a disconnected fragment of a portion can be as small as a single primary particle. For such a small fragment it is not correct to neglect cohesion (and Brownian motion) during its settling, while assuming that cohesion inside larger fragments is strong enough to assure their stability. However, one may argue that the asymptotic structure is governed by the larger fragments so that the wrong dynamics for the smallest ones should not matter so much. Alternatively one may envisage a more subtle physical situation, where a freshly formed contact is only weakly cohesive, but cohesion becomes stronger the longer a contact exists. In this case our model would be justified for the smallest fragments, as well. 